SPECT showed hyperperfusion in left occipito-parietal, left lateral temporal, and left inferior frontal regions. Brain MRI was normal. Two varieties of reading epilepsy are differentiated, jaw jerk variant and posterior variant with visual symptoms. (Gavaret M, Guedj E, Koessler L, et al. Reading epilepsy from the dominant temporo-occipital region. J Neurol Neurosurg Psychiatry July 2010;81:710-715). (Dr Martine Gavaret, Service de Neurophysiologie Clinique, Hopital de la Timone, 264 rueSaint Pierre, Marseille 13005, France. E-mail: martine.gavaret@ap-hm.fr).

COMMENT. Bickford RG and colleagues at Mayo Clinic were the first to describe reading epilepsy as a new syndrome (Trans Am Neurol Assoc 1956;81:100102). Seizures were precipitated by reading difficult material, and the cortical or lexical aspects of the seizure provocation were stressed by Bickford and others. In a report from University of Ottawa, Canada, a 24-year-old woman experienced jaw jerking while reading aloud material of medium or high linguistic complexity (Christie $\mathrm{S}$ et al. Epilepsia 1988;29L288-293). These authors found several factors might be involved in seizure precipitation while reading. These included saccadic eye movements, articulation, in addition to difficulty of reading content, and no factor acted solely as the critical stimulus. In a further Mayo Clinic report of 20 patients diagnosed between 1949 and 1989, age at onset ranged from 10 to 46 years (median 17 years). Seizures were myoclonic, involving orofacial and jaw muscles, and generalized tonic-clonic. EEG showed generalized spike or spike and wave discharges in 15 patients and left hemisphere discharges in 5. Seizures responded to valproic acid. (Radhakrishnan K, Silbert PL, Klass DW. Brain 1995;118:75-89).

In an editorial, Koepp MJ of the UK emphasizes that although jaw jerks are the hall mark of reading epilepsy, many other reading-induced ictal symptoms are described: absence seizure, abrupt loss of consciousness, paroxysmal alexia or dyslexia, and prolonged stuttering. Dr Koepp advocates more case studies, similar to that of Gavaret et al, to determine the localization and mechanism of reading epilepsy, in contrast to the current focus on statistical analysis of large random samples in epilepsy research.

\title{
SPEECH AND LANGUAGE DYSFUNCTION AND FOCAL EPILEPTIFORM ACTIVITY
}

The medical history, EEG recordings, and developmental milestones of 19 children with speech and language dysfunction, and focal epileptiform activity were analyzed for prognostic risk factors in a study at University of Gothenburg, Sweden. Three patterns of language development were observed at follow-up: 1) late start and continued slow development (possible Landau-Kleffner syndrome (LKS) or epileptic language disorder (ELD); 2) late start and deterioration/regression (probable LKS); and 3) normal start and later regression/deterioration (definite LKS). Seizures (complex partial, generalized tonic-clonic, absence, perioral, and eye-lid myoclonic) occurred in 15 patients but were rare. Age at onset was $2-9$ years. Response to AEDs (carbamazepine, etc) was poor, whereas corticosteroids were effective in 8 of 9 cases. Six (32\%) had a family history of seizures. All but one had normal CT or MRI. SPECT showed hypoperfusion in left temporal region. Family history was positive for speech and language delay or reading and writing disability. Early general development was normal 
in $14(74 \%)$. Early language development was late in $13(68 \%)$, and normal in 6; later deterioration occurred in $9(47 \%)$. EEG spike/spike-and-wave activity was temporal in 12 $(63 \%)$ and parietal, frontal or central location in 7. CSWS (continuous spike and wave during slow wave sleep) was found in 7 (37\%), and 7 had excess epileptiform activity during sleep. At age $9-25$ years at follow-up, EEG was normal in 10 and showed epileptiform activity in 9. Cognitive ability was average in 7, and variably impaired in 12 . Long-term speech and language function is unfavorable in $50 \%$ of participants and not more favorable in any one of the three LKS patterns (possible, probable, and definite). In addition to language dysfunction, patients with LKS at follow-up have auditory dysfunction, oral-motor dysfunction, stuttering, and cognitive dysfunction. Persisting epileptiform activity and a family history of seizures are risk factors for a poor prognosis. Further study of antiepileptic treatment is recommended. (Selassie G R-H, Hedstrom A, Viggedal G, Jennische M, Kyllerman M. Speech, language, and cognitive dysfunction in children with focal epileptiform activity: a follow-up study. Epilepsy Behav Jüly 2010:18:267-275). (Respond: Dr Gunilla R-H Selassie. E-mail: gunilla.rejno-habteselassie@vgregion.se).

COMMENT. Three patterns of language development are found in children with sleep-activated epileptiform activity and language disorder (Landau-Kleffner syndrome or epileptic language disorder). Long-term prognosis is unfavorable in $50 \%$, and risk factors for a poor outcome include persistent epileptiform activity and a family history of seizures.

Functional connectivity, language impairment, and localization-related epilepsy. Activation maps and functional connectivity networks were studied by fMRI in 34 adults (mean age 40 years) with cryptogenic localization related epilepsy and language impairment and compared to 20 healthy controls. Activation maps did not differ between patients and controls, but patients with epilepsy had significantly lower functional connectivity in left frontal temporal networks during text reading and on the wordfluency test. Impaired language performance in epilepsy patients is associated with loss of functional connectivity in cognitive language networks. (Vlooswijk MCG et al. Neurology August 3, 2010;75:395-402).

\section{SEIZURE DISORDERS}

\section{VIDEO-EEG STUDY OF SEIZURE PREVALENCE IN COMATOSE PICU PATIENTS}

The prevalence of epileptic seizures (clinical and subclinical) in 100 consecutive comatose children aged 2 months to 17 years in the pediatric ICU was determined using continuous video-EEG ( $\mathrm{v}$-EEE) monitoring, in a prospective study at The Royal Children's Hospital, Melbourne, Australia. Half of the monitored patients had primary brain disorders, including traumatic brain injury, hypoxic ischemic encephalopathy, and status epilepticus; half had brain dysfunction secondary to systemic sepsis or organ failure. Epileptic seizures were identified in 7 patients, 6 having a history of epilepsy and a seizure witnessed immediately prior to the v-EEG. Median length of ICU stay was 5 